\title{
Facial Augmentation by Intra-Oral Delivery of Autologous Fat
}

\section{Libby R. Copeland-Halperin ${ }^{1}$, Michelle Copeland ${ }^{2}$}

${ }^{1}$ Department of Surgery, Inova Fairfax, Falls Church, VA; ${ }^{2}$ Division of Plastic and Reconstructive Surgery, Icahn School of Medicine at Mount Sinai, New York, NY, USA
No potential conflict of interest relevant to this article was reported.
Background As harvesting techniques have improved the viability of transposed adipose tissue, autologous fat transfer is increasingly utilized for facial augmentation. Conventional techniques involve placing harvested fat deep in subcutaneous tissue through skin punctures. Trans-oral fat injections at the buccal and labial sulci and through the mucosa of the oral commissures obviate external scars. We report experience with this technique for augmentation of the cheeks, zygomatic arch, nasolabial folds, upper and lower lips, and chin in 130 patients over a 14-year period.

Methods We report an observational case series of 147 procedures performed in 130 patients ( 118 women and 12 men, 19-69 years of age) in which 10 to $60 \mathrm{~mL}$ autologous fat supernatant harvested from the hips, buttocks, or abdomen were injected through the oral mucosa for augmentation. Single preoperative doses of antibiotic and methylprednisolone were administered in all cases. Fat frozen and banked during initial treatments was used for additional augmentation 3 to 6 months after initial treatments in $46 \%$ of cases. Outcomes were assessed clinically with physical exams and photographs. Results Mucosal puncture wounds healed uneventfully. Most patients tolerated the procedures well and recovered rapidly compared to historical experience with conventional percutaneous techniques. One patient developed a sterile seroma 3 weeks after facial augmentation that responded to needle aspiration.

Conclusions Transmucosal, intra-oral autologous fat grafting was associated with minimal visible scarring, swelling, or infection at the recipient site. This technique may be considered an alternative to percutaneous transfer for proximate peri-oral facial augmentation.

Keywords Cosmetic techniques, Reconstructive surgical procedures, Rejuvenation, Subcutaneous fat, Tissue transplantation

\section{INTRODUCTION}

Changes in the deep subcutaneous tissues of the face are intrinsic to aging and most often associated with infrastructure collapse [1]. Indeed, atrophy of the facial form is a hallmark of the aging face

Received: Aug 30, 2015 Revised: Nov 2, 2015 Accepted: Nov 4, 2015 Correspondence: Michelle Copeland Division of Plastic and Reconstructive Surgery, Icahn School of Medicine at Mount Sinai 1001 5th Avenue, New York, NY 10028, USA. E-mail: mcopeland@drcopeland.com

Copyright @ 2016 The Korean Society for Aesthetic Plastic Surgery.

This is an Open Access article distributed under the terms of the Creative Commons Attribution Non-Commercial License (http://creativecommons.org/licenses/by-nc/3.0/) which permits unrestricted non-commercial use, distribution, and reproduction in any medium, provided the original work is properly cited. www.e-aaps.org
[2]. As fat harvesting techniques have improved, public confidence in synthetic materials (e.g., silicone, Teflon ${ }^{\circledR}$ [Dupont, Wilmington, DE, USA]), and foreign tissues (collagen) has waned, and problems with absorption and long-term survival of translocated fat have diminished. Autologous fat transplants-injections of extracted fatty tissue from the abdomen, hips, buttocks, or thighs-are widely used to augment facial structure [3-7]. Despite the introduction of safer, temporary, hyaluronic fillers, patients often prefer injections of autologous fat over foreign material, and greater volumes can be transferred to enhance multiple areas at lower cost than temporary fillers. Furthermore, improved storage techniques allow for supplementary fat transfer without the need for repeated harvesting. The frequency of fat injection procedures in the United States rose 215\% between 1992 and 2000 and 87.8\% from 1997 to 2012 [8]. We de- 
scribe our experience with 147 intra-oral procedures in $130 \mathrm{pa}-$ tients for augmentation of the cheeks, zygomatic arch (ZA), nasolabial folds, chin, and upper and lower lips.

\section{METHODS}

The technique involved injection of fat through intra-oral punctures at the buccal and labial sulci and through the mucosa of the oral commissures. Between 10 and 60 milliliters of supernatant fat harvested from the hips, buttocks, or abdomen, were injected using 10-gauge needles under local anesthesia. A single dose of an antibiotic, generally cephalexin, was given preoperatively, and a single dose of methylprednisolone was administered intra-operatively in all cases.

The donor sites were selected for cosmetic benefit of fat removal, usually the outer thighs, buttocks, or abdomen, since fat from these regions has high lipogenic activity [2,9]. Patients were positioned supine, prepped with benzalkonium chloride solution, and draped. Mild preoperative sedation with oral diazepam was supplemented with nitrous oxide administered via nasal cannula throughout the procedure. Xylocaine ${ }^{\circledR}$ (AstraZeneca LP, Wilmington, DE, USA) $(1 \%)$ and epinephrine $(1: 100,000)$ were injected at the donor site in $400 \mathrm{~mL}$ lactated Ringer’s solution with lidocaine (Marcaine ${ }^{\circledR}$; Hospira, Lake Forest, IL, USA). A 1-mm incision was made with a \#15 blade. Fat was then aspirated through the incision with a 60 $\mathrm{mL}$ syringe using a size-3 Becker Toomey cannula. A total of 100 $\mathrm{mL}$ of fat was harvested, mixed, cleansed with saline without centrifugation, and transferred to $10-\mathrm{mL}$ syringes. The donor site incision was closed with 5-0 Vicry ${ }^{\circledR}$ sutures. Cleaned fat was stored for later use in a standard freezer compartment in $10-\mathrm{mL}$ sterile syringes and sterile plastic bags.

The recipient sites-generally the nasolabial folds, cheeks, lips, and chin-were anesthetized intra-orally with $1 \%$ Xylocaine $^{\circledR}$ (AstraZeneca) with epinephrine (1:100,000) using 25-gauge needles, and fat was injected using 10-gauge needles. Intra-oral incisions 1 $\mathrm{mm}$ in length were made on the right and left corners of the commissures of the lip, and the needle was advanced through the subcutaneous tissue while fat was gently injected. Injections in the cheeks and chin were made through a 1-mm buccal or labial incision above the periosteum, delivering enough fat to achieve approximately $30 \%$ over-correction (Fig. 1). Approximately 30 to $40 \mathrm{~mL}$ was injected to the cheeks, nasolabial folds, lips, and chin during the initial procedures. Typically, an additional 20 to $30 \mathrm{~mL}$ were injected during subsequent sessions 3 to 6 months later. Postoperatively, durability of fat grafting, infection, and scarring were assessed clinically with physical exams and photographs.

\section{RESULTS}

The mean age of the 118 women and 12 men was 50 years (range

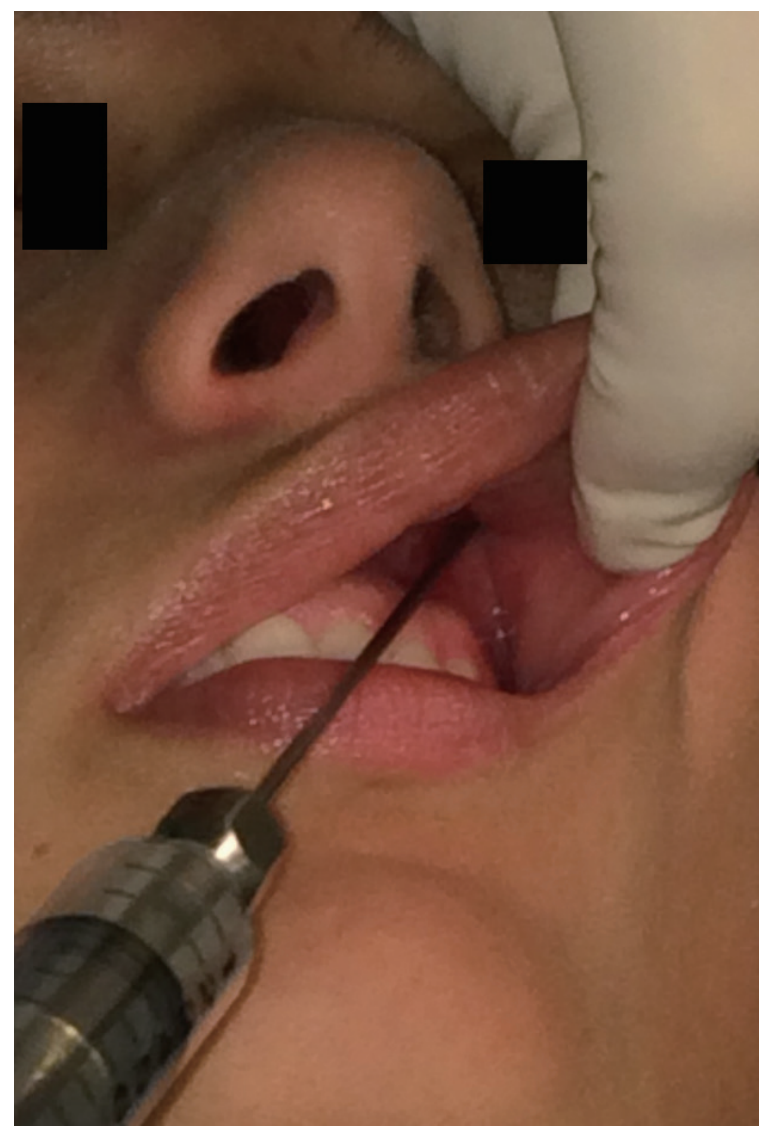

Fig. 1. Intra-operative injection technique. Intra-operative injection technique through buccal sulcus for cheek augmentation.

19-69 years). One hundred percent of patients had augmentation of the nasolabial folds and cheeks, 50\% also had lip augmentation, and $30 \%$ also had augmentation of the chin. Fat frozen and banked at the time of initial treatments was used for additional augmentation 3 to 6 months after initial treatment in $46 \%$ of cases. We observed no visible scars, and regional swelling due to the volume of injected liquid was rapidly absorbed, so the appearance of overcorrection subsided after 2 weeks (Fig. 2). Mucosal puncture wounds healed uneventfully, and most patients tolerated the procedures well and recovered rapidly compared to historical experience with conventional percutaneous techniques. One patient developed a sterile seroma 3 weeks after facial augmentation that responded to needle aspiration. The overall infection rate was the same for men and women and across each injection site. Long-term durability depended on initial over-correction, similar to the conventional approach. This technique was employed to augment the cheeks, ZA, nasolabial folds, upper and lower lips, and chin (Fig. 2 and 3).

\section{DISCUSSION}

Fat grafting for facial augmentation was first described in 1893 when 

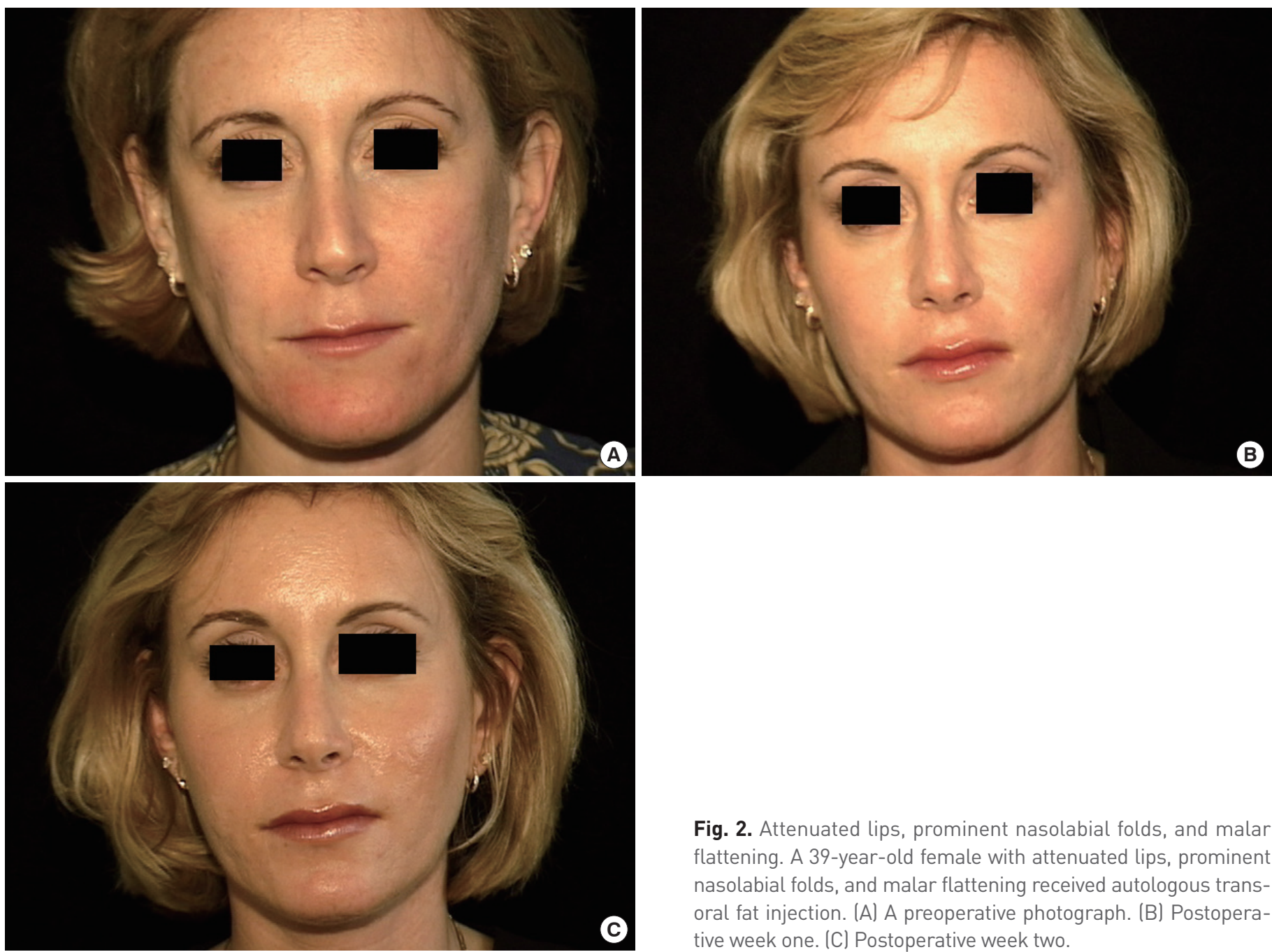

Fig. 2. Attenuated lips, prominent nasolabial folds, and malar flattening. A 39-year-old female with attenuated lips, prominent nasolabial folds, and malar flattening received autologous transoral fat injection. (A) A preoperative photograph. (B) Postoperative week one. (C) Postoperative week two.
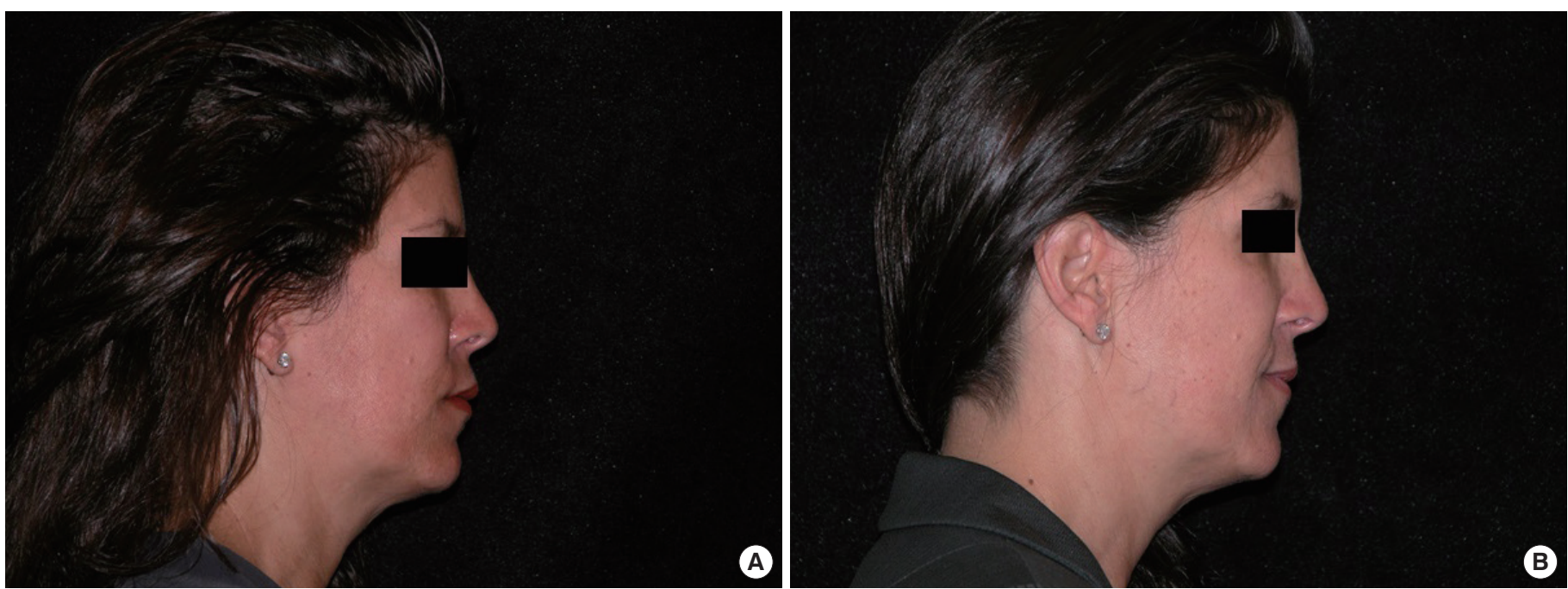

Fig. 3. Malar flattening, visible nasolabial folds, and attenuation of lips. A 50-year-old female with malar flattening, visible nasolabial folds, and attenuation of the lips received autologous trans-oral fat injection. (A) A preoperative photograph. (B) Postoperative month 6. 
Neuber [10] reconstructed facial defects by transferring $1-\mathrm{cm}$ pieces of fat from the upper arm. Injection of autologous fat to correct post-rhinoplasty deformities was first reported by Brunnings in 1911, who injected small pieces of fat using a hypodermic needle and syringe [11]. Fat transplantation was revived after the introduction of liposuction in 1977 [12], and Fournier coined the term "liposculpture" to describe a procedure by which adipose tissue was removed with liposuction and selectively injected to correct irregularities in other areas [12].

Conventionally, percutaneous autologous fat transfer relocates harvested fat deep into subcutaneous tissue using a syringe and large-core needle, or cannula attached to a suction device. Advantages include ease of performance and supplementation, minimal induction of immune-mediated inflammation, relatively low cost, avoidance of secondary defects, rapid recovery, and minimal risk. Major drawbacks are reabsorption, which can be lessened by new techniques and overcome by repeated treatments, and bruising and redness of the recipient site, due mainly to puncture wounds $[8,10]$.

In the 1990s, Coleman [3] further refined the harvesting, purification, and injection techniques. The use of small-caliber cannulas (3-4 mm), so threads of transplanted adipose tissue do not exceed $3 \mathrm{~mm}$ in diameter, reduces fat necrosis and improves fat survival. Recent reports describe transfer of injected fat particles as small as $2 \mathrm{~mm}$ [13]. Applying a low level of negative pressure during aspiration minimizes trauma to adipocytes, and rinsing aspired fat with normal saline prolongs implant survival [4]. These procedural advances have been associated with greater viability of transposed autologous fat.

Previous studies described the use of autologous fat injection grafting for cosmetic facial augmentation [14,15], but these studies did not involve an intra-oral approach. We identified one report of intra-oral fat injection for correction of lip hemangiomas [16]. A common criticism of intra-oral fat transfer is the belief that oral injection is associated with a greater risk of infection than use of the percutaneous approach. However, the precise incidence of infection using a trans-oral or transmucosal route of injection has not been reported in the literature to support this claim. We did not observe an increased rate of infection using this technique, mucosal puncture wounds healed uneventfully, recovery was rapid, and swelling, bruising, redness, and scarring at the recipient site were negligible inside the mouth. These findings are consistent with use of the transmucosal route in many other surgeries, such as synthetic malar and chin implants, and maxillary and mandibular osteotomies with plate and wire fixation.

The majority of the patients we encountered tolerated the procedure well, and 46\% chose additional augmentation 3 to 6 months later, using fat banked and frozen during the first session. This duration of fat stability is consistent with reports of extra-oral fat grafting $[9,17]$. The decision to receive additional reinjections after the initial fat augmentation was based on the patient's own subjective assessment and preferences as to the amount of augmentation desired and was independent of the initial injection site. As with the conventional technique, long-term persistence of augmentation depended on the initial over-correction. Clinically applicable objective methods for assessing the longevity of fat injections are lacking. While some studies use magnetic resonance imaging or other formal imaging techniques, this is often not practical or cost-effective in clinical practice. We judged the duration of fat stability based on physical exam and photographs taken at each visit, similar to previous studies [2,14].

Although this is a small case series and more formal controlled studies are necessary, our study demonstrates that transmucosal, intra-oral delivery of autologous fat for proximate peri-oral facial augmentation can reduce visible scarring and swelling at the recipient site. Furthermore, this technique was associated with minimal risk of infection. Transmucosal intra-oral fat transfer should be considered an alternative to percutaneous transfer for peri-oral augmentation.

\section{PATIENT CONSENT}

Patients provided written consent for the use of their images.

\section{REFERENCES}

1. Gonzalez-Ulloa M, Flores ES. Senility of the face: basic study to understand its causes and effects. Plast Reconstr Surg 1965;36:239-46.

2. Donofrio LM. Structural autologous lipoaugmentation: a pan-facial technique. Dermatol Surg 2000;26:1129-34.

3. Coleman SR. Long-term survival of fat transplants: controlled demonstrations. Aesthetic Plast Surg 1995;19:421-5.

4. Coleman SR. Structural fat grafts: the ideal filler? Clin Plast Surg 2001; 28:111-9.

5. Moscona R, Ullman Y, Har-Shai Y, et al. Free-fat injections for the correction of hemifacial atrophy. Plast Reconstr Surg 1989;84:501-7.

6. Niechajev I, Sevćuk O. Long-term results of fat transplantation: clinical and histologic studies. Plast Reconstr Surg 1994;94:496-506.

7. Guerrerosantos J, Gonzalez-Mendoza A, Masmela Y, et al. Long-term survival of free fat grafts in muscle: an experimental study in rats. Aesthetic Plast Surg 1996;20:403-8.

8. American Society of Plastic Surgeons. ASPRS resources. Arlington Heights, IL: American Society of Plastic Surgeons; 2001 [cited 2015 Jan 1]. Available from http://www.plasticsurgery.org/.

9. Lithell H, Boberg J. The lipoprotein-lipase activity of adipose tissue from different sites in obese women and relationship to cell size. Int J Obes 1978;2:47-52.

10. Neuber GA. Fettransplantation. Chir Kongr Verhandl Dtsch Gesellsch Chir 1893;22:66-8.

11. Ersek RA, Chang P, Salisbury MA. Lipo layering of autologous fat: an improved technique with promising results. Plast Reconstr Surg 1998; 
101:820-6.

12. Mandrekas AD, Zambacos GJ, Kittas C. Cyst formation after fat injection. Plast Reconstr Surg 1998;102:1708-9.

13. Mashiko T, Yoshimura K. How does fat survive and remodel after grafting? Clin Plast Surg 2015;42:181-90.

14. Marten TJ, Elyassnia D. Fat grafting in facial rejuvenation. Clin Plast Surg 2015;42:219-52.
15. Coleman SR. Facial augmentation with structural fat grafting. Clin Plast Surg 2006;33:567-77.

16. Yin J, Li H, Yin N, et al. Autologous fat grafting in lip reconstruction following hemangioma treatment. J Craniofac Surg 2013;24:346-9.

17. Kaufman MR, Miller TA, Huang C, et al. Autologous fat transfer for facial recontouring: is there science behind the art? Plast Reconstr Surg 2007;119:2287-96. 